\title{
Wave height forecasting to improve off-shore access and maintenance scheduling
}

\author{
I. Dinwoodie, V. M. Catterson, and D. McMillan \\ Institute for Energy and Environment, University of Strathclyde, \\ Glasgow, United Kingdom. \\ Email: v.m.catterson@strath.ac.uk
}

\begin{abstract}
This paper presents research into modelling and predicting wave heights based on historical data. Wave height is one of the key criteria for allowing access to off-shore wind turbines for maintenance. Better tools for predicting wave height will allow more accurate identification of suitable "weather windows" in which access vessels can be dispatched to site. This in turn improves the ability to schedule maintenance, reducing costs related to vessel dispatch and recall due to unexpected wave patterns.
\end{abstract}

The paper outlines the data available for wave height modelling. Through data mining, different modelling approaches are identified and compared. The advantages and disadvantages of each approach, and their accuracies for a given site implementation, are discussed.

\section{INTRODUCTION}

Within Europe, development of offshore wind farms has gained significant momentum in recent years with a particular focus on the North Sea. The first round of projects in the UK were considered 'near-shore', built in relatively shallow water depths of less than $20 \mathrm{~m}$ and within $20 \mathrm{~km}$ of shore. However, current and future offshore wind farms are increasing in distance to shore and water depth due to planning constrictions and favourable wind conditions. Consequently, the number and size of individual turbines in future wind farms will be larger to reduce overall cost of energy. This will result in maintenance becoming more challenging logistically, and critical for mitigating lost revenue associated with downtime of a large turbine.

This paper investigates methods of wave height modelling with the aim of predicting future wave heights accurately enough to assist with maintenance planning. While physical modelling is possible [1], the creation of a physical wave model requires detailed information about the location and geography. This paper takes a data mining approach to modelling, resulting in the implementation, test, and comparison of autoregressive (AR) models and artificial neural network (ANN) models at varying lookahead time steps.

While both approaches were found to perform similarly using the RMS error metric, the results were analysed in light of the types of vessel and their wave height restrictions which are used for maintenance. Key wave heights of $1.5 \mathrm{~m}$ and $3.5 \mathrm{~m}$ were studied separately, showing that the AR models can capture the shape of wave extremes more accurately than the ANN models, which capture shape only up to a 12 hour lookahead window.
TABLE I. TYPICAL COSTS AND RESTRICTIONS ON ACCESS[2][3]

\begin{tabular}{|c|c|c|c|}
\hline Vehicle & Day Rate $(£)$ & Main restrictions & Height Limit \\
\hline $\begin{array}{c}\text { Access } \\
\text { transfer } \\
\text { boat }\end{array}$ & 1750 & $\begin{array}{c}\text { Operability } \\
\text { limited by } \\
\text { wave height }\end{array}$ & $1.5 \mathrm{~m}$ \\
\hline $\begin{array}{c}\text { Field } \\
\text { support } \\
\text { vessel }\end{array}$ & 9500 & $\begin{array}{c}\text { Limit depending } \\
\text { on turbine } \\
\text { access method }\end{array}$ & $1.5-3 \mathrm{~m}$ \\
\hline Helicopter & $\begin{array}{c}9600 \text { (small) }- \\
28800 \text { (large) }\end{array}$ & $\begin{array}{c}\text { Limited by health and } \\
\text { safety for sea rescue }\end{array}$ & $3.5 \mathrm{~m}$ \\
\hline $\begin{array}{c}\text { Mobile } \\
\text { Jack-up } \\
\text { vessel }\end{array}$ & $\begin{array}{c}100000-250000 \\
\text { dependent } \\
\text { on water depth }\end{array}$ & $\begin{array}{c}\text { Jack and } \\
\text { movement limited } \\
\text { by sea state }\end{array}$ & $2 \mathrm{~m}$ \\
\hline
\end{tabular}

\section{OFF-SHORE MAINTENANCE}

Maintenance of offshore wind farms is restricted by the access constraints of service vehicles. Currently, the predominant means of accessing an offshore turbine has been using an access transfer boat. Larger failures require more specialised access either through field support vessels, helicopter, or mobile jack-up. Table I identifies when each is typically used, typical day rate cost associated with each, and how wave climate affects accessibility.

All maintenance actions for offshore wind are directly or indirectly dependant on wave climate. Minimising the hire duration of maintenance vehicles is critical to controlling O\&M costs. Improved methods for predicting the access windows based on wave climate are therefore essential for the industry moving forward.

\section{DAtA Mining FOR WAVE Height PREDiction}

Given the need for accurate models for wave height prediction, this work aimed to compare and contrast different approaches to data-driven modelling, and draw conclusions about the suitability of such techniques. The data mining steps taken were:

- Familiarisation with the data and domain,

- Data cleaning and reduction,

- Technique selection and testing,

- Comparison of results.

Due to the inherent harsh operating environment and remote location, gathering wave climate data is expensive and it is difficult to obtain significant continuous sections of data, particularly in locations representative of offshore wind farms. One solution to this lack of high quality data would be to use hindcast data based on wind and satellite observations. 
However, creating a model based on inferred data can be considered poor practice, raising questions about the accuracy of the inference and hence the model.

One source of measured data with an adequate duration in the North Sea was identified as the FINO 1 offshore research platform [4] located $45 \mathrm{~km}$ off the German coast in the zone marked for development of future offshore wind farms. As well as having sufficient wave data to test the predictive capabilities of the described modelling approaches (spanning multiple years), there is concurrent wind data which can be used for future work linking wave and wind access.

The FINO 1 research platform has been collecting wave height data continuously since 2004 , although gaps exist within the data set due to the poor reliability of wave buoys used to carry out the measurements. In order to carry out predictions of wave height based on historical data it was necessary to fill these gaps. Any measurements missing for less than four time steps (corresponding to less than 12 hours, since measurements are taken at 3 hour intervals) were filled using a cubic interpolation. Gaps longer than this were replaced with the corresponding averaged value at the same time step across all other years in order to create a complete time series covering seven years.

An alternative to statistical modelling for wave height forecasts is physics-based models of wave creation, propagation and dispersion. These models have been improved radically in the past decades and include so-called 3rd generation approaches, which attempt to capture nonlinear interactions of wind input and energy dissipation of waves. These are comprehensively reviewed by [1]. Other models attempt to characterise near shore waves specifically utilising approaches tuned to coastal processes [5]. These approaches explicitly model the physics of the wave formation and interaction process, and as a result tend to be more computationally heavy, and require more data, compared with statistical methods [6]. Furthermore, for short term forecasting (0-10 hours) which will often be used for wind farm accessibility, statistical forecasts tend to outperform those from physical models [6].

\section{A. Technique Selection}

It was decided to focus on data-driven models instead of physics-based models, given the advantages discussed above. However, there are still a great many such techniques which may be applicable. For this initial study, two commonly-used techniques were chosen: auto-regression (AR), and artificial neural networks (ANNs) [6]. These techniques are different enough that there is scope for different predictive capabilities, yet they can be trained and tested on the same data.

The data was partitioned into two versions of training and test sets, as described in Table II, one covering one year of data (2004) and the second covering two (2004 and 2005). The remainder of the dataset, up to January 2011, was used as test data in each case.

It was thought necessary to include at least one full year of data in the training set, as the data exhibits a strong pattern of seasonality. For the AR model in particular, this seasonality should be removed to improve the model's predictive capabilities, and this is easier to achieve with a full example of the
TABLE II. PARTITIONING OF TRAINING DATA SETS

\begin{tabular}{|c|c|c|}
\hline Training data years & No. Samples & Test data years \\
\hline 2004 & 2928 & $2005-2010$ inclusive \\
\hline 2004 and 2005 & 5848 & $2006-2010$ inclusive \\
\hline
\end{tabular}

seasonal effect. In addition, it was anticipated that two years of training data would show a greater range of wave behaviour than one year alone.

Both techniques were used to train models which predict future wave height, on the basis of four sequential historical readings. These readings were taken at three hour intervals, which means the input data represents 12 hours of historical wave heights.

For each technique, models were trained with varying lookahead windows from one time step up to 10 . The one time step model takes four sequential wave heights and predicts the next (giving 3 hours of lookahead), with the two time step model predicting wave height in 6 hours, up to the 10 time step model predicting wave height in 30 hours' time. The following sections describe in detail the training of each set of models.

1) AR model: The general form of an $\mathrm{AR}$ model as described in [7] is shown in Eq. 1, normalised with respect to the mean, $\mu$ and in terms of model parameters $\varphi_{i}$ and Gaussian white noise term $\varepsilon_{t}$.

$$
X_{t}=\mu+\varepsilon_{t}+\sum_{i=1}^{p} \varphi_{i}\left(X_{t-i}-\mu\right)
$$

Eq. 1 is valid for a stationary process due to the presence of $\varepsilon_{t}$. This is not applicable to wave data and consequently data must be pre-processed before the modelling approach can be applied. A Box-Cox transformation and removal of seasonal average is applied to the data [7] in order that the data approximates a normal distribution. For wave data, the Box-Cox shape parameter, $\lambda=0$ and the transformation takes the form of Eq. 2 where $Y_{t}$ is the transformed time series and $\hat{\mu}_{l n\left(H s_{t}\right)}$ is a Fourier Series estimate of the time series of the logarithm means [8].

$$
Y_{t}=T\left(H s_{t}\right)=\ln \left(H s_{t}\right)-\hat{\mu}_{l n\left(H s_{t}\right)}
$$

This process is demonstrated in Figure 1 showing the probability density function (pdf) of a sample data set before and after transformation.

For the AR model itself, determination of the model coefficients was carried out using the MATLAB System Identification Toolbox by iteratively solving the Yule-Walker equations. Simulation of the look-ahead value predictions were performed using the predict routine with fitted coefficients within the same toolbox.

2) ANN model: Since an ANN can handle non-linearity, there was no need to perform the Box-Cox transformation to remove the seasonality. Raw wave height data was used as training data directly.

A standard multi-layer perceptron (MLP) architecture was chosen, with four input nodes for the historical wave heights, and the output node containing a linear activation function (as 

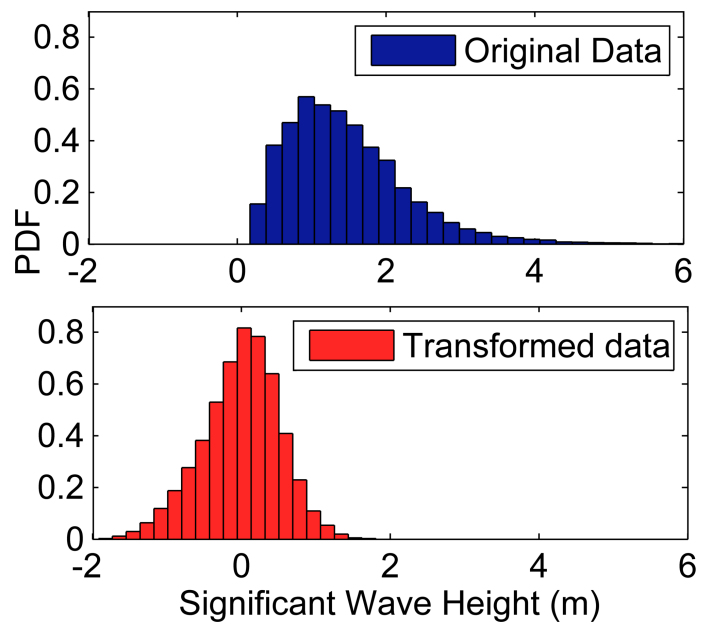

Fig. 1. The effect of removing seasonality and transforming input data

TABLE III. EFFECT OF HIDDEN NODES ON RMS ERROR

\begin{tabular}{|c|c|}
\hline Hidden nodes & RMS error \\
\hline 2 & 0.2422 \\
\hline 3 & 0.2448 \\
\hline 4 & 0.2445 \\
\hline 5 & 0.2463 \\
\hline 6 & 0.2454 \\
\hline 7 & 0.2496 \\
\hline 8 & 0.2569 \\
\hline 9 & 0.2725 \\
\hline 10 & 0.2700 \\
\hline
\end{tabular}

recommended for a regression model). The number of hidden nodes was found through experimentation, ranging from two up to and including 10.

Model training was performed using the R nnet library, with weight decay of 0.0005 and the maximum number of iterations increased to 1000 . Model predictions were generated using the nnet.predict routine.

The RMS error on test data was taken as the metric for assessing the appropriate number of hidden nodes. For this analysis, the training data set was the 2004 data, and a lookahead prediction of one time step was used. Three separate runs were performed for each number of hidden nodes and on each training set. The lowest RMS error results are shown in Table III. This shows that the highest accuracy is achieved with two hidden nodes, giving a 4-2-1 MLP architecture.

3) Discussion of the models: The choice of four input terms was driven largely by the auto-regressive model analysis. The auto and partial correlation function plots of input data (Figure 2), and the classification information in Table IV suggest that four time steps $(\mathrm{AR}(4))$ captures sufficient time dependency while remaining computationally efficient.

This can be linked to the physical processes driving wave height, which are very different between far offshore and near shore locations. A very clear diurnal trend is observed in wind speed data in coastal locations which drives wave heights near shore [9]. Further offshore these diurnal patterns are not present [10], however, there is a strong seasonal component which can be considered deterministic. It is to remove this seasonal effect that the Box-Cox transform is applied, before fitting the AR model.
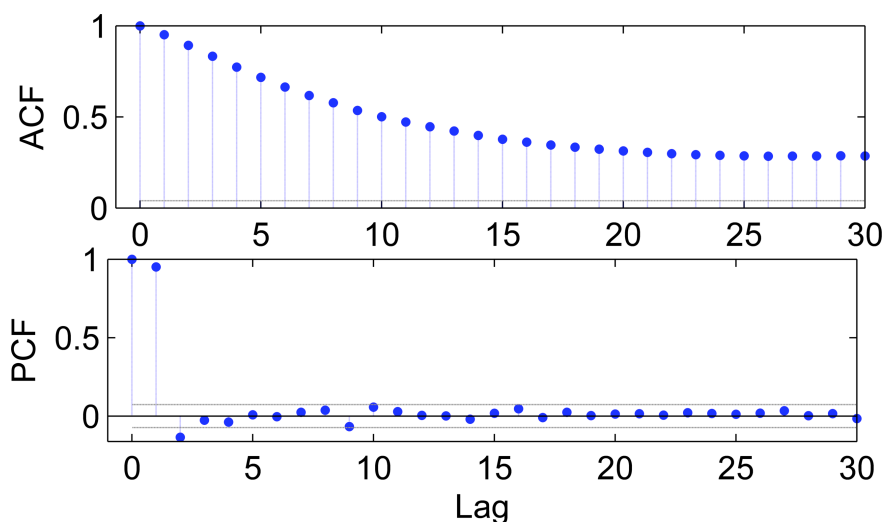

Fig. 2. Auto and partial correlation functions (ACF and PCF)

TABle IV. Auto Regressive Model Order ClassificAtion [11]

\begin{tabular}{|c|c|c|}
\hline Model & ACF Description & PCF Significant Terms \\
\hline AR(1) & $\begin{array}{c}\text { Exponential or } \\
\text { oscillatory decay }\end{array}$ & $\Phi_{k k} \approx 0$ for $k>1$ \\
\hline AR(2) & $\begin{array}{c}\text { Exponential or } \\
\text { sinusoidal decay }\end{array}$ & $\Phi_{k k} \approx 0$ for $k>2$ \\
\hline AR(p) & $\begin{array}{c}\text { Exponential and/or } \\
\text { sinusoidal decay }\end{array}$ & $\Phi_{k k} \approx 0$ for $k>p$ \\
\hline
\end{tabular}

For a direct comparison with the ANN, the input parameters were kept the same for both techniques.

\section{B. Results}

The RMS error of each model is shown in Figure 3. As may be expected, the further into the future the model is predicting, the higher the RMS error, regardless of the modelling technique or training data set chosen. It can be seen that both the AR and ANN approaches perform very similarly when evaluated on the RMS error metric, with the AR models very slightly outperforming the ANNs. The RMS error at one lookahead step ( 3 hours) for all models is under $0.25 \mathrm{~m}$, rising to $0.7 \mathrm{~m}$ at 10 lookahead steps (30 hours).

Figure 3 also shows a persistance line, which is the accuracy of prediction made simply by assuming the wave height persists from its value $n$ time steps in the past. For example, for the one time step lookahead, the previous recorded wave height is assumed to persist for the next measurement. For the 10 time step lookahead, the previous wave height it assumed to predict the wave height in 10 time steps' time. While the persistance model matched the capabilities of the others at one and two time step lookahead, all AR and ANN models outperformed the persistance model from three steps and upwards.

The effect of different training data sets was different from the authors' initial hypothesis. It was expected that a longer training period would result in more accurate models, reflected by a lower RMS error. The RMS error is in fact greater for the longer training data set, although the effect is very slight.

The training period did have an effect on the ANN results. While the lowest RMS errors for each training data set are very similar, the shorter training period resulted in more of a spread of ANN accuracy across different training runs and different numbers of hidden nodes; that is, some of the ANNs performed particularly poorly with less training data, while others were still able to learn the regression model as accurately as the AR 


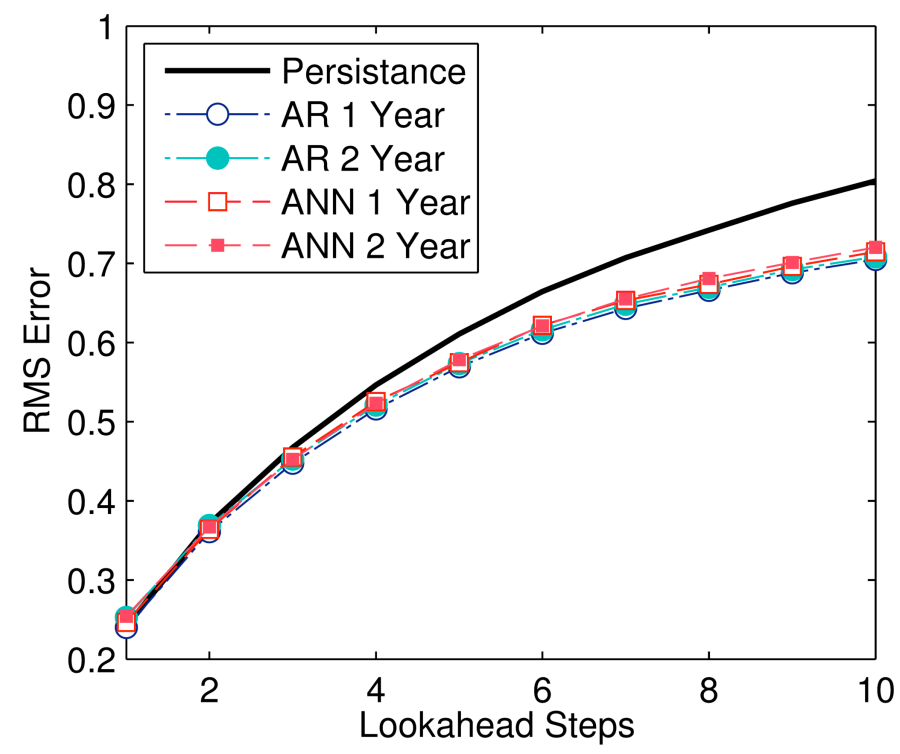

Fig. 3. RMS error of each model for different lookahead time steps

TABLE V. RMS ERRORS ABOVE KEY WAVE HEIGHTS (1 YEAR TRAINING DATA)

\begin{tabular}{|c|c|c|c|c|}
\hline Look- & \multicolumn{2}{|c|}{ ANN RMS errors } & \multicolumn{2}{c|}{ AR RMS errors } \\
ahead & $>1.5 \mathrm{~m}$ & $>3.5$ & $>1.5 \mathrm{~m}$ & $>3.5$ \\
\hline 1 & 0.4899 & 1.0856 & 0.3162 & 0.6995 \\
2 & 0.5967 & 1.3926 & 0.4918 & 1.1564 \\
3 & 0.6898 & 1.6920 & 0.6154 & 1.4721 \\
4 & 0.7599 & 1.9238 & 0.7109 & 1.7342 \\
5 & 0.8110 & 2.0816 & 0.7845 & 1.9420 \\
6 & 0.8551 & 2.2321 & 0.8438 & 2.1114 \\
7 & 0.8812 & 2.3136 & 0.8868 & 2.2486 \\
8 & 0.9069 & 2.3782 & 0.9197 & 2.3509 \\
9 & 0.9266 & 2.4428 & 0.9494 & 2.4272 \\
10 & 0.9426 & 2.4957 & 0.9720 & 2.4925 \\
\hline
\end{tabular}

technique. This highlights the variability of the ANN training process, and the need for multiple runs to identify a "true" ANN accuracy.

With regard to Table I, particularly notable wave heights are $1.5 \mathrm{~m}$, which limits access transfer boats; and $3.5 \mathrm{~m}$, which is the firm upper limit on access due to accessibility for rescue helicopters. Therefore, the models were examined for their performance above these thresholds.

As anticipated, the more extreme wave heights are predicted with poorer accuracy, and the increasing lookahead window only compounds the errors (see Table V). While the accuracy for waves above $1.5 \mathrm{~m}$ is still under half a meter (for a single lookahead time step), this rises to over $1 \mathrm{~m}$ of RMS error for waves above $3.5 \mathrm{~m}$ in height. At the furthest lookahead of 30 hours, the RMS error for waves over $3.5 \mathrm{~m}$ in height is almost $2.5 \mathrm{~m}$, which is so large in comparison to the predicted height that the prediction is essentially meaningless.

Another way of visualizing the performance of the AR models is given in Figure 4. This plot shows the actual wave height data in black, followed by the AR models' predictions of successive timesteps as colored traces. An ideal set of models would show the colored traces exactly replicating the shape of the actual data, spaced out by single timesteps. Instead of this ideal pattern, each AR model can be characterized as replicating the general shape of the trace, but not correctly capturing the extreme wave height values.

As an example, the largest peak between data points 8270 and 8300 sees the true wave height exceed $8 \mathrm{~m}$, after two lower peaks nearer $2 \mathrm{~m}$ and $4 \mathrm{~m}$. The blue trace for the one timestep prediction matches the two lower peak values, but predicts a less extreme value for the largest peak. Each successive timestep prediction places the three peaks at the correct times; however the predicted wave height becomes less and less extreme in its peak value.

This smoothing effect is largely due to the choice of an AR(4) model, where only four previous time steps are impacting the subsequent value. Increasing the auto-regressive order does not improve this performance as there is insufficient structure in the data as identified in Figure 2. The model fails to predict extreme peaks and troughs in the data as they are the result of consecutive increases in values, which a Gaussian noise term will not predict.

In contrast, the ANN model does not capture the shape of large deviations as well as the AR model (see Figure 5). From one to four time steps of prediction the shape is preserved relatively well, but at intervals greater than this the averaging effect dominates. The range of predicted wave heights is curtailed, with all predictions lying closer to the mean.

In particular, the peak between data points 8270 and 8300 is present in the one, two, three, and four timestep models, but the shape of the peak differs quite significantly between the one and two timestep models (the blue trace is a broader peak than the thinner red trace). None of the ANN predictions exceeds $6 \mathrm{~m}$, for a true wave height above $8 \mathrm{~m}$. In addition, the dark red of the nine timestep model shows a definite peak at 8295 , with no corresponding peak in the black data trace. This is very different from the AR models, which only peak with a corresponding peak in the true wave height.

Improvements in both models, in order to capture the observed extreme behaviour, are an area for future research.

\section{CONCLUSIONS}

The need for applied research in the field of wave height forecasting for offshore wind accessibility and maintenance will only increase in future years as more offshore assets are installed, and those assets move through their life cycle. The economic value of increased accuracy becomes clear as turbine capacities increase, leading to large lost revenues for unplanned outages. The need to plan robustly taking weather conditions into account will be a key tool in controlling O\&M spend for offshore wind in the future.

Future work will study differences of wave regimes in different water depths, consider the cost/benefit case for accurate forecasting in more detail, and examine possible use of regime switching [12] or threshold time series models [13] as well as more advanced neural networks and copula models more commonly used in wind forecasting [14], and evidence combination techniques for integrating the output of multiple models [15]. Such research will primarily help wind farm owners operate their plant more efficiently, with lower costs. 


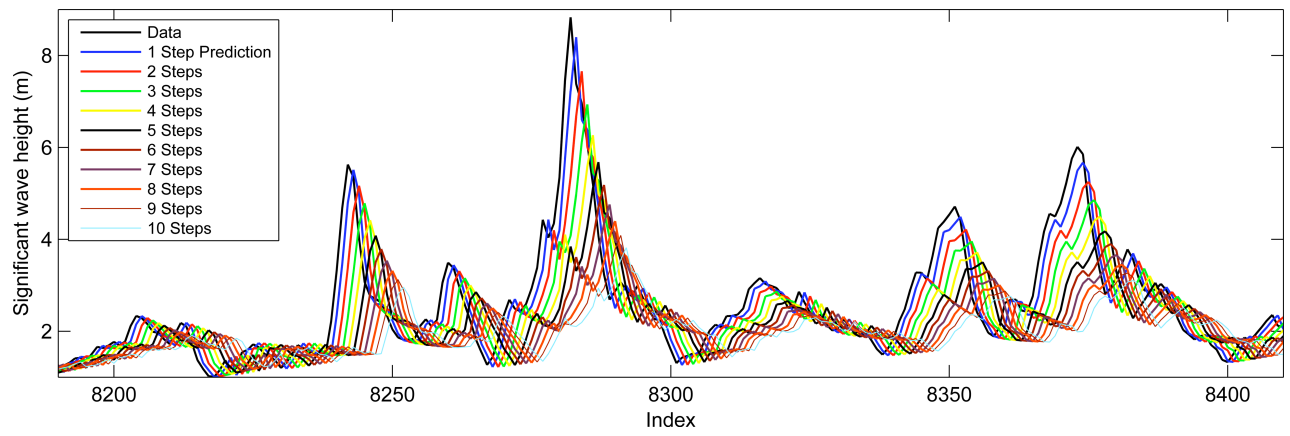

Fig. 4. Part of the testing time series with AR model predictions overlayed. The shape is well-captured, but the range decreases with increased lookahead.

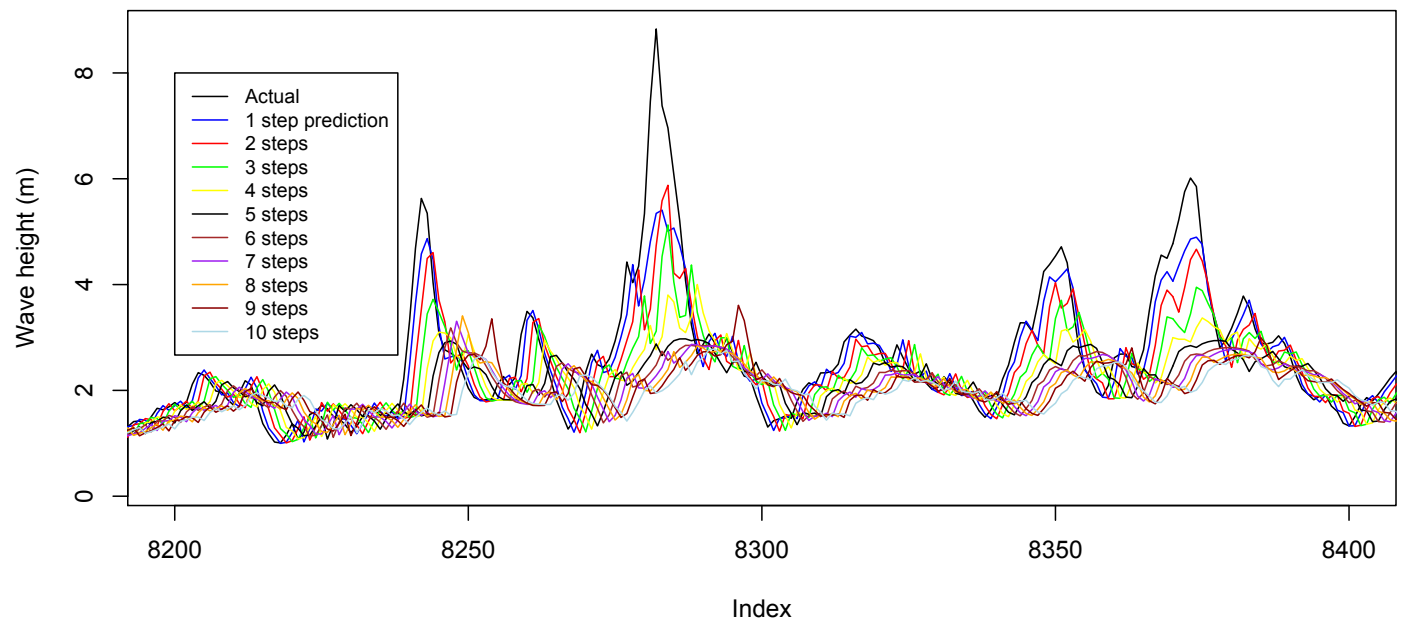

Fig. 5. Part of the testing time series with ANN model predictions overlayed. The shape is somewhat well-captured up to four steps of lookahead.

\section{ACKNOWLEDGEMENT}

For data from the FINO project, we thank the BMU (Bundesministerium fuer Umwelt, Federal Ministry for the Environment, Nature Conservation and Nuclear Safety) and the PTJ (Projekttraeger Juelich, project executing organisation).

This work was supported by EPSRC Grant EP/G037728/1, Centre for Doctoral Training in Wind Energy Systems.

\section{REFERENCES}

[1] P. A. E. M. Janssen, "Progress in ocean wave forecasting," Journal of Computational Physics, vol. 227, no. 7, pp. 3572-3594, Mar. 2008.

[2] P. Tavner, Offshore Wind Turbines: Reliability, Availability and Maintenance. IET, 2012.

[3] I. Dinwoodie and D. McMillan, "Heavy Lift Vessel Strategy Analysis for Offshore Wind," in European Wind Energy Association (EWEA), Feb. 2013.

[4] FINO, "Forschungsplattformen in Nord- und Ostsee," 2012, available http://www.fino-offshore.de/.

[5] H. C. M. Smith and D. E. Reeve, "Modelling studies of the sensitivity of the shoreline wave climate to the proposed wave farm development off the north coast of Cornwall," Ocean Engineering, vol. 34, no. 5-6, pp. 884-901, 2011.

[6] G. Reikard, P. Pinson, and J. Bidlot, "Forecasting ocean wave energyThe ECMWF wave model with time-series methods," Ocean Engineering, vol. 38, no. 10, pp. 1089-1099, 2011.
[7] G. Box, G. Jenkins, and G. Reinsel, Time Series Analysis: Forecasting and Control. Wiley, 2008.

[8] C. Cunha and C. Guedes Soares, "On the choice of data transformation for modelling time series of significant wave height," Ocean Engineering, vol. 26, no. 6, pp. 489 - 506, 1999.

[9] D. Hill, K. Bell, D. McMillan, and D. Infield, "Application of autoregressive models to UK wind speed data for power system impact studies," IEEE Trans. Sustainable Energy, vol. 3, no. 1, Jan. 2012.

[10] I. A. Dinwoodie, D. McMillan, and F. Quail, "Offshore wind turbine operation and maintenance analysis using a time domain meteo-ocean modelling approach," in ASME Turbo Expo 2012, 2012.

[11] X. Wang and J. McDonald, Modern power system planning. McGrawHill, 1994.

[12] P. Pinson, L. Christensen, H. Madsen, P. Sørensen, M. Donovan, and L. Jensen, "Regime-switching modelling of the fluctuations of offshore wind generation," Journal of Wind Engineering and Industrial Aerodynamics, vol. 96, no. 12, pp. 2327-2347, 2008.

[13] R. Killick, I. Eckley, and P. Jonathan, "Efficient Detection Of Multiple Changepoints Within An Oceanographic Time Series," in Proceedings of the 58th Session of ISI, 2011.

[14] B. Stephen, S. Galloway, D. McMillan, D. Hill, and D. Infield, "A Copula Model of Wind Turbine Performance," IEEE Trans. Power Systems, vol. 26, no. 2, pp. 965-966, May 2011.

[15] V. M. Catterson and S. D. J. McArthur, "Using Evidence Combination for Transformer Defect Diagnosis," Int. J. Innovations in Energy Sys. \& Power (IJESP), vol. 1, Nov. 2006. 